\title{
Cancer, obesity, and diabetes: TKIs exert multiple effects on glucose homeostasis
}

\section{Minglin Lin and Junfei Jin}

Cancer, obesity, and type II diabetes mellitus (T2DM) are becoming increasingly prevalent worldwide; a greater understanding of the links between these medical conditions is urgently needed in order to identify novel targets for treatment. In a Review published in the February 2017 issue of this distinguished journal (Cancer, obesity, diabetes, and antidiabetic drugs: is the fog clearing? Nat. Rev. Clin. Oncol. 14, 82-99; 2017) ${ }^{1}$, Klil-Drori et al. summarize the current evidence regarding the relationships between obesity, T2DM, and cancer. We completely agree with the authors' statements regarding the influence of various medications used to treat diabetes on cancer development and/or outcomes. Moreover, we appreciate the clear evidence presented by the authors that, conversely, some anticancer treatments can perturb glucose homeostasis and, therefore, lead to or exacerbate hyperglycaemia and T2DM. This Review neglects, however, the potential effects of tyrosine-kinase inhibitors (TKIs) that are used routinely in the treatment of cancer on glycaemic control.

TKIs are now used successfully in the treatment of many malignancies. Many of these agents target signalling pathways that influence cellular glucose metabolism, and increasing amounts of data demonstrate that TKI therapy can result in either elevated or decreased blood glucose levels, depending on the agent used and the clinical context ${ }^{2,3}$
(TABLE 1). In some settings, hyperglycaemia has been reported as a common adverse effect of TKI therapy ${ }^{4}$. Generally, however, TKIs contribute to lower glucose level and, thus, improve glycaemic control. In one study ${ }^{5}$, the hypoglycaemic effects of imatinib, dasatinib, sorafenib, and sunitinib occurred in both diabetic and nondiabetic patients, but the exact underlying mechanisms remain to be determined. Importantly, this effect might actually be beneficial for patients with T2DM by enabling them to discontinue antidiabetic drugs; in the aforementioned study ${ }^{5}$, eight of the 17 diabetic patients (47\%) attained a level of glycaemic control with TKI therapy that enabled them to discontinue their antidiabetic medications, including insulin in some patients.

Of note, some TKIs (for instance, imatinib and sunitinib) have been associated with both hyperglycaemia and hypoglycaemia ${ }^{6,7}$, adding an additional layer of complexity. Indeed, certain TKIs can exert totally opposite glycaemic effects in different types of cancer. In a case report involving a patient with a relapsed and refractory abdominal solitary fibrous tumour, sorafenib treatment - given in the context of an initial misdiagnosis of refractory gastrointestinal stromal tumour - was effective in controlling persistent non-islet-cell tumourinduced hypoglycaemia (NICTH $)^{8}$. NICTH is caused by overexpression of insulin-like
Table 1 | Effect of TKIs approved for the treatment of cancer on glucose homeostasis

\begin{tabular}{|c|c|c|}
\hline TKI & Approved indications & Effect on glucose levels \\
\hline Imatinib & CML, CEL, ALL, and GIST & $\begin{array}{l}\text { - Hyperglycaemic effect }{ }^{7} \\
\text { - Hypoglycaemic effect }{ }^{10}\end{array}$ \\
\hline Nilotinib & CML & Hyperglycaemic effect ${ }^{4}$ \\
\hline Dasatinib & CML and ALL & Hypoglycaemic effect ${ }^{11}$ \\
\hline Gefitinib & NSCLC & Hyperglycaemic effect ${ }^{12}$ \\
\hline Erlotinib & NSCLC, gastic cancer, and PDAC & Hypoglycaemic effect ${ }^{13}$ \\
\hline Sorafenib & $\mathrm{RCC}$ and $\mathrm{HCC}$ & Hypoglycaemic effect ${ }^{5}$ \\
\hline Sunitinib & RCC, GIST, and PNET & $\begin{array}{l}\text { - Hyperglycaemic effect } t^{14} \\
\text { - Hypoglycaemic effect }{ }^{15}\end{array}$ \\
\hline \multicolumn{3}{|c|}{$\begin{array}{l}\text { ALL, acute lymphoblastic leukaemia; CEL, chronic eosinophilic leukaemia; CML, chronic myeloid } \\
\text { leukaemia; GIST, gastrointestinal stromal tumour; HCC, hepatocellular carcinoma; NSCLC, non-small-cell } \\
\text { lung carcinoma; PDAC, pancreatic ductal adenocarcinoma; PNET, pancreatic neuroendocrine tumour; } \\
\text { RCC, renal-cell carcinoma. }\end{array}$} \\
\hline
\end{tabular}

growth factor II (IGF-II) by tumour cells; therefore, the observed effect of sorafenib on hypoglycaemia was probably due, in part, to tumour-cell killing - as supported by evidence of intratumoural necrosis on CT scans ${ }^{8}$. Nevertheless, the glycaemic control might also have been partially attributable to inhibition by sorafenib of receptor-tyrosine kinases that mediate glucose metabolism. By contrast, in addition to the hypoglycaemic effects of sorafenib when used in approved indications ${ }^{5}$, this agent has also been associated with triggering of recurrent nonhyperinsulinaemic hypoglycaemia (in a wax and wane pattern) in a patient with haemangiopericytoma who had never previously experienced hypoglycaemia9. The mechanisms contributing to hypoglycaemia in this case might also have been associated with NICTH because the patient's serum IGF-II levels were slightly elevated; however, the possibility that hypoglycaemia resulted from the activity of sorafenib itself and/or the effects of this agent on tumour burden cannot be excluded ${ }^{9}$.

In summary, TKIs exert markedly different effects on glucose homeostasis according to the particular TKI or the type of tumour involved. Thus, research investigating the relationships between these anticancer agents, diabetes, and cancer is urgently needed.

Minglin Lin and Junfei Jin are at the Laboratory of Hepatobiliary and Pancreatic Surgery, Affiliated Hospital of Guilin Medical University, 15 Lequn Road, Guilin, 541001, Guangxi, China.

Correspondence to J.J. changliangzijin@163.com

Klil-Drori, A. J., Azoulay, L. \& Pollak, M. N. Cancer, obesity, diabetes, and antidiabetic drugs: is the fog clearing? Nat. Rev. Clin. Oncol. 14, 85-99 (2017).

2. Shlomai, G., Neel, B., LeRoith, D. \& Gallagher, E. J. Type 2 diabetes mellitus and cancer: the role of pharmacotherapy. J. Clin. Oncol. 34, 4261-4269 (2016).

3. Malek, R. \& Davis, S. N. Tyrosine kinase inhibitors under investigation for the treatment of type II diabetes. Expert Opin. Investig. Drugs 25, 287-296 (2016).

4. Saglio, G. et al. Nilotinib versus imatinib for newly diagnosed chronic myeloid leukemia. N. Engl. J. Med. 362, 2251-2259 (2010).

5. Agostino, N. M. et al. Effect of the tyrosine kinase inhibitors (sunitinib, sorafenib, dasatinib, and imatinib) on blood glucose levels in diabetic and nondiabetic patients in general clinical practice. J. Oncol. Pharm. Pract. 17, 197-202 (2011).

6. Verges, B., Walter, T. \& Cariou, B. Endocrine side effects of anti-cancer drugs: effects of anti-cancer targeted therapies on lipid and glucose metabolism. Eur. J. Endocrinol. 170, R43-R55 (2014).

7. Lodish, M. B. \& Stratakis, C. A. Endocrine side effects of broad-acting kinase inhibitors. Endocr. Relat. Cancer 17, R233-R244 (2010).

8. Le Jeune, S. et al. Refractory hypoglycemia controlled by sorafenib in solitary fibrous tumor. J. Clin. Oncol. 31, e118-e121 (2013).

9. Lee, S. W. et al. Recurrent hypoglycemia triggered by sorafenib therapy in a patient with hemangiopericytoma. Endocrinol. Metab. (Seoul) 29, 202-205 (2014).

10. Veneri, D., Franchini, M. \& Bonora, E. Imatinib and regression of type 2 diabetes. N. Engl. J. Med. 352 1049-1050 (2005). 


\section{CORRESPONDENCE}

11. Ono, K. et al. Rapid amelioration of hyperglycemia facilitated by dasatinib in a chronic myeloid leukemia patient with type 2 diabetes mellitus. Intern. Med. $\mathbf{5 1}$ 2763-2766 (2012).

12. Simon, G. R. et al. Phase 2 trial of docetaxel and gefitinib in the first-line treatment of patients with advanced nonsmall-cell lung cancer (NSCLC) who are 70 years of age or older. Cancer 112, 2021-2029

(2008).
13. Costa, D. B. \& Huberman, M. S. Improvement of type 2 diabetes in a lung cancer patient treated with erlotinib. Diabetes Care 29, 1711 (2006).

14. Motzer, R. J. et al. Sunitinib versus interferon alfa in metastatic renal-cell carcinoma. N. Engl. J. Med. 356, 115-124 (2007).

15. Oh, J. J. et al. Impact of sunitinib treatment on blood glucose levels in patients with metastatic renal cell carcinoma. Jpn J. Clin. Oncol. 42, 314-317 (2012).
Acknowledgements

The work of the J.J. is supported by grants from the National Natural Science Foundation of China (81572738), the Natural Science Foundation of Guangxi (2015GXNSFEA139003), and the Lijiang Scholar Award.

Competing interests statement

The authors declare no competing interests. 